\title{
Unusual Presentation of Large B Cell Lymphoma- Bone and Stomach- Treated with Autologous Transplantation
}

\author{
Bokyung Kim, M.D. ${ }^{1}$, Sung Yong Oh, M.D. ${ }^{1}$, Suee Lee, M.D. ${ }^{1}$, Hyuk-Chan Kwon, M.D. ${ }^{1}$, Sung-Hyun \\ Kim, M.D. ${ }^{1}$, Sook Hee Hong, M.D. ${ }^{2}$, Sung-Soo Kim, M.D. ${ }^{3}$ and Hyo-Jin Kim, M.D. ${ }^{1}$ \\ Departments of ${ }^{1}$ Internal Medicine, ${ }^{2}$ Pathology, ${ }^{3}$ Orthopedic Surgery, Dong-A University College of Medicine, Busan, Korea
}

Extranodal presentation of diffuse large $B$ cell lymphoma (DLBL) is frequently observed in the gastrointestinal tract, CNS, bone, testes and liver. However, the simultaneous detection of multiple extranodal involvement at presentation is quite an uncommon occurrence. In this study, we report on a patient with an uncommon presentation of DLBL, and he had symptoms of left knee joint pain and hematemesis, characterized by bone and stomach involvement. Computed tomography and fluorodeoxyglucose-positron emission tomography (FDG-PET) scanning revealed a rapid, extensive spread to the bones and soft tissues. Subsequent histopathological examination verified the bony and gastric CD20-positive DLBL locali-

\section{INTRODUCTION}

Diffuse large B cell lymphoma (DLBL) constitutes approximately $30 \%$ of all non-Hodgkin's lymphomas (NHLs). This typically presents with a rapidly enlarging symptomatic mass, which is generally the consequence of nodal enlargement. Extranodal disease with involvements of tissues other than the lymph nodes, spleen, Waldeyer's ring and thymus is quite commonly observed among DLBL patients (1).

The origin of primary extranodal NHLs can frequently be traced to one specific organ system or site, with the most common extranodal sites being the stomach, skin and small intestine, yet the breast, thyroid, lung, heart, liver, genitourinary tract, adrenals and bones can also be primarily affected $(2 \sim 4)$. However, a heterogeneous group of NHLs that includes DLBL, Burkitt's lymphoma and mucosa-associated lymphoma tissue (MALT) lymphoma can involve multiple extranodal sites at presentation (5). In this study, we describe a patient who evidenced an unusual presentation of DLBL that simultaneously involved the femur, tibia, fibula and stomach. Fluorodeoxyglucose-positron emission tomography (FDG-PET) scanning revealed a rapid and extensive spread to the nodes and bones.

Correspondence: Hyo-Jin Kim, Department of Internal Medicine, Dong-A University College of Medicine, 3-1, Dongdaesin-dong, Seo-gu, Busan 602-715, Korea. (Tel) 82-51-240-2951, (Fax) 82-51-240-2951, (E-mail) kimhj@dau.ac.kr.

Received October 24, 2007, Accepted November 12, 2007 zation. We diagnosed this case as DLBL of stage IV with an international prognostic index of 3 , and classified him into the high intermediate risk group. This patient was treated via chemotherapy with an R-CHOP regimen. After achieving a partial response, the patient received autologous peripheral blood stem cell transplantation. The patient attained partial remission, as shown on the FDG-PET scan, and he displayed improvement of his left femur pain. (Cancer Res Treat. 2007;39:181-184)

Key Words: Extranodal, Diffuse large B-cell lymphoma, Stomach, Bone

A subsequent histopathological examination confirmed CD 20-positive DLBL.

\section{CASE REPORT}

A 45 year-old man was admitted to the orthopedic department due to pain in the left knee that had developed 1 year previously. The patient had no remarkable personal medical history or familial history.

During the clinical examination, the patient appeared well; his blood pressure was $110 / 70 \mathrm{mmHg}$ and his pulse was 80 beats/min. The lungs were clear on auscultation. The patient's abdomen was soft and non-tender; no hepatomegaly or splenomegaly was detected. Swelling and tenderness were detected at the medial side of the left knee and a $4 \times 4 \mathrm{~cm}$ mass was observed. The chest radiographic examination revealed no abnormalities. Left knee magnetic resonance imaging (MRI) was compatible with lymphoma involvement in the distal femur, proximal tibia and fibula (Fig. 1A). We then performed an open biopsy of the left distal femur under spinal anesthesia.

While waiting for the results, the patient visited the emergency room due to his hematemesis and melena. His blood pressure was $110 / 70 \mathrm{mmHg}$ and his pulse was 118 beats/min. The laboratory findings indicated anemia $(\mathrm{Hb}: 87 \mathrm{~g} / \mathrm{L}$, RBCs: $3.23 \times 10^{12} / \mathrm{L}, \mathrm{MCV}: 75.9 \mathrm{fL}$ ) with increased serum lactate dehydrogenase (LDH: $1055 \mathrm{IU} / \mathrm{L})$. All the other laboratory findings were nonspecific.

Emergency endoscopy was conducted and we detected huge ulcerative lesions in the stomach body and posterior wall of the angle of the stomach. Fortunately, we noted no active bleeding 

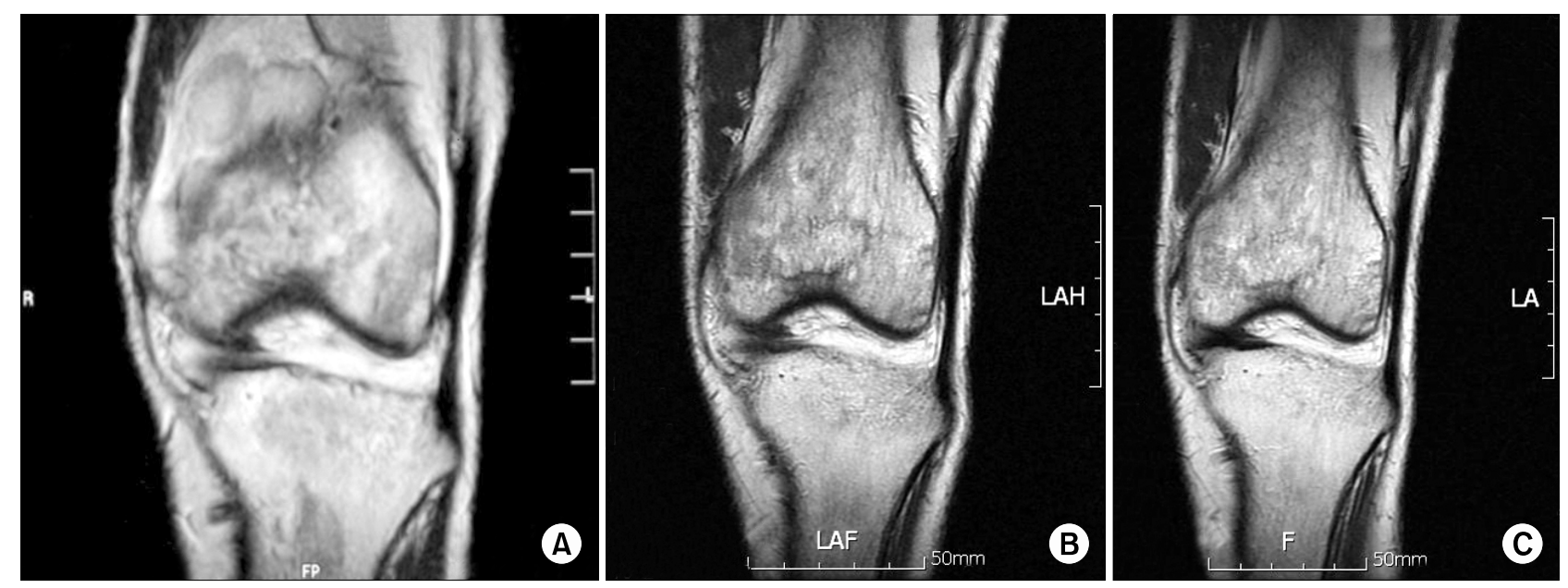

Fig. 1. Left knee magnetic resonance imaging series. (A) Left knee magnetic resonance imaging (MRI) initially shows lymphoma involvement of the distal femur, proximal tibia, fibula etc. and extension to the suprapatellar fat pad. (B) Left knee MRI after 3 cycles of chemotherapy. (C) Left knee MRI after 6 cycles of chemotherapy.
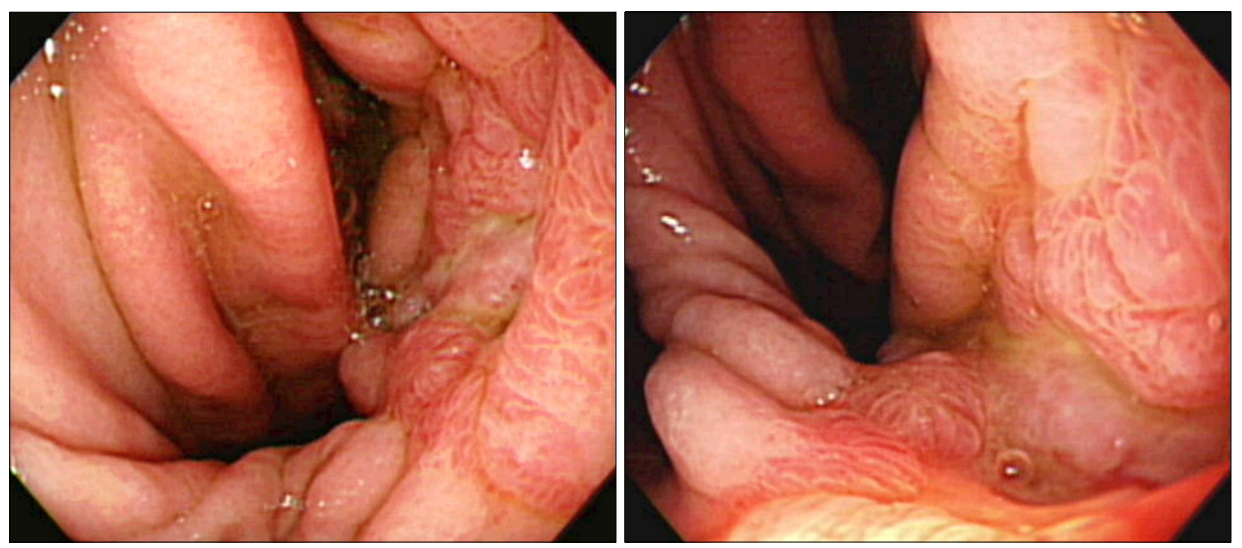

Fig. 2. Endoscopic findings of the patient. Note the gastric ulcer in the lower body and posterior wall of the angle of the stomach.

and the patient's anemia was corrected after transfusion of packed cells. After 1 week, we observed on the follow-up endoscopy that the gastric ulcer was in a healing state (Fig. 2), so we conducted a multiple biopsy there. The Campylobacterlike organism (CLO) test was negative.

On the histologic examination, the neoplasm had a diffuse growth pattern and this consisted of medium and large-sized cells with focal aggregations of large uniform cells. The immunophenotypes of the malignant cells were CD $20\left(^{+}\right)$, LCA (+), CD $99(+)$, TdT (-), UCHL-1 (-) and CD $68(+)$, suggesting a B-cell origin. Therefore, the immuno-histopathological examination of the left distal femur and stomach confirmed CD20-positive DLBL (Fig. 3).

We also assessed the results of a CT scan, bone scan and FDG-PET scan. A right supraclavicular lymph node of approximately $1 \mathrm{~cm}$ was suggested on the chest CT scan. The CT scan of the abdomen and pelvis revealed a small hepatic cyst (approximately $1 \mathrm{~cm}$ ) and a gallbladder polyp (approximately $0.5 \mathrm{~cm}$ ), but no evidence of lymphadenopathy. No hepatomegaly or splenomegaly was detected on the CT scan. A bone scan with technectium-99m diphosphonate identified multiple uptake sites in the femur, tibia, right fibula and left ulna. The FDG-PET scan indicated multiple bony involvement in the right scapula (max standardized uptake value (SUV): 3.0), right clavicle (max SUV: 6.0), and both the femur and fibula (max SUV: 19.0) with soft tissue extension (max SUV: 20.7). Multiple hypermetabolic lymph node lesions were also observed in the right upper internal jugular (max SUV: 2.7), right supraclavicular (max SUV: 3.0), and right interpectoral (max SUV: 2.6) areas (Fig. 4). The stomach showed evidence of normal glucose metabolism.

Lymphomatous involvement was observed on neither the pelvic bone marrow aspiration nor the biopsy. Consequently, we diagnosed this case as DLBL of stage IV with an international prognostic index (IPI) of 3, and we classified the patient into the high intermediate risk group.

The patient was treated via chemotherapy with an R-CHOP regimen (rituximab $375 \mathrm{mg} / \mathrm{m}^{2}$ - cyclophosphamide $750 \mathrm{mg} / \mathrm{m}^{2}$ - doxorubicin $50 \mathrm{mg} / \mathrm{m}^{2}$ - vincristine $1.4 \mathrm{mg} / \mathrm{m}^{2}$ on day 1 , and prednisolone $100 \mathrm{mg}$ /day on days $1 \sim 5$, every 21 days). The re-evaluation, which included the left knee MRI, evidenced good partial remission after three and six cycles (Fig. 1B, C). 

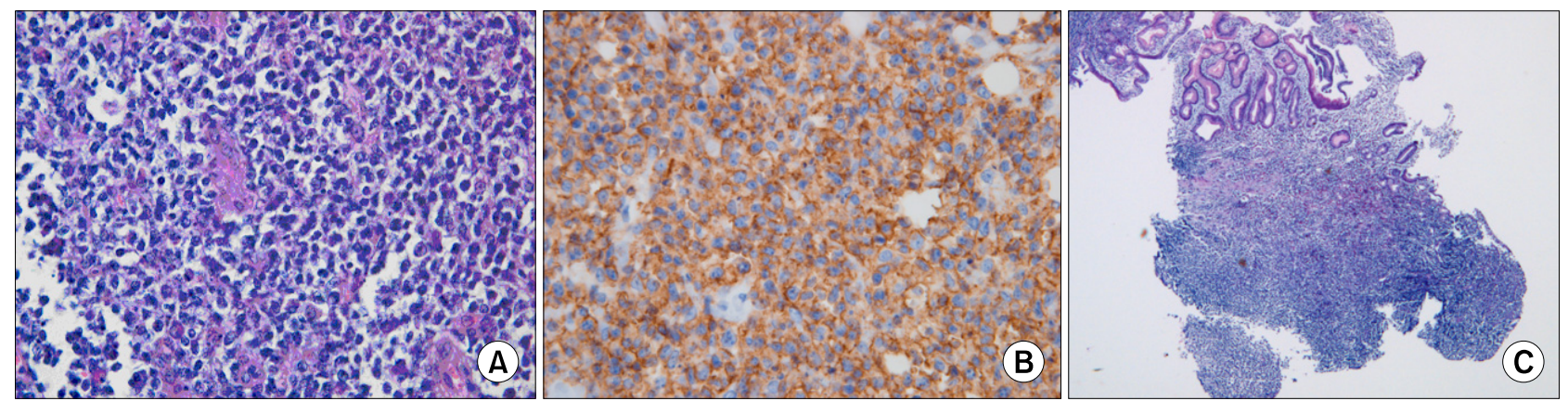

Fig. 3. Pathologic findings of the bone and stomach. (A) Haematoxylin-eosin staining showing the bone tissue with diffuse infiltration by large lymphomatous cells $(\times 400)$. (B) Lymphomatous cells expressing CD 20 antigen $(\times 400)$ in the bone tissue. (C) Infiltration of the gastric mucosal and submucocal layer by dense neoplastic lymphoid cells $(H \& E, \times 200)$.
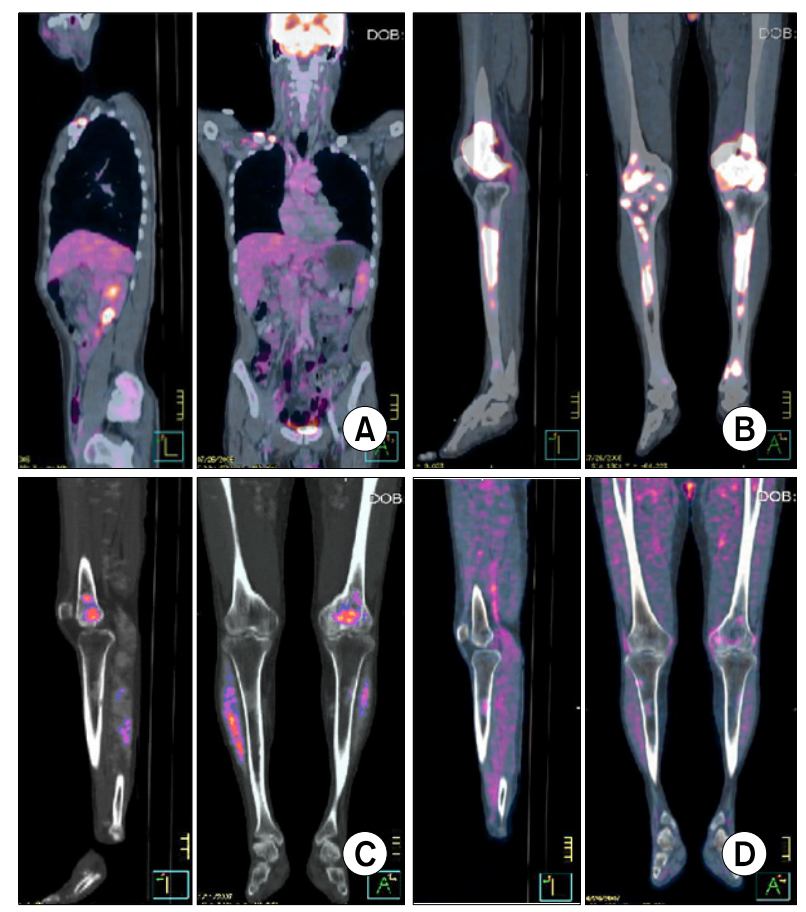

Fig. 4. The FDG-PET scanning of the patient. (A, B) The FDGPET scanning revealed multiple bony involvements of both the femur and fibula (max SUV: 19.0) with soft tissue extension (max SUV: 20.7). Multiple hypermetabolic lesions were observed in the right upper internal jugular (max SUV: 2.7), right supraclavicular (max SUV: 3.0), and right interpectoral (max SUV: 2.6) lymph nodes. (C) The follow-up FDG-PET scanning after 6 cycles of chemotherapy shows a markedly improved condition, but hypermetabolic lesions persisted in the left femur and both tibias (max SUV: 1.8). (D) The follow-up FDG-PET scanning after PBSCT shows a slight improvement in the condition of the left femur (max SUV: 1.1).

FDG-PET scanning after six cycles revealed marked improvement, but hypermetabolic lesions persisted in the left femur and both tibias (max SUV: 1.8) (Fig. 4C). The other hypermetabolic lesions, including those in the right scapula as well as multiple lymph nodes, were normalized. The patient underwent an additional three cycles of chemotherapy, and he then received autologous peripheral blood stem cell transplantation (PBSCT). High-dose cyclophosphamide $\left(4 \mathrm{~g} / \mathrm{m}^{2}\right)$ followed by granulocyte-colony-stimulating factor (G-CSF) was utilized for PBSC mobilization, and a sufficient number of CD34+ cells $\left(3.3 \times 10^{6} / \mathrm{kg}\right)$ were collected. The conditioning chemotherapy consisted of busulfan $\left(130 \mathrm{mg} / \mathrm{m}^{2}, \mathrm{D}-8,-7,-6\right.$ and -5$)$, etoposide $(20 \mathrm{mg} / \mathrm{kg}, \mathrm{D}-4)$, and cyclophosphamide $(50 \mathrm{mg} / \mathrm{kg}, \mathrm{D}-3$ and D-2). We achieved partial remission as evidenced on the FDG-PET scan, which showed a slightly improved condition of the left femur (max SUV: 1.1) (Fig. 4D).

\section{DISCUSSION}

NHL is the term utilized for a group of disorders that originate from the malignant transformation of lymphocytes and this involves either the lymph nodes or extranodal sites. Extranodal lymphomas may comprise $24 \sim 48 \%$ of the NHL cases, and the incidence of these lymphomas appears to have increased over the past few decades (6). Although there have been previous reports regarding primary extranodal NHL at various sites, and especially GI-NHL, there remain many questions regarding the clinicopathological features and treatment outcomes of these patients. These factors are well known in the context of Burkitt's, angiotropic and angiocentric lymphomas (7), but the multifocal extranodal appearance of a variety of histological subtypes has not yet been fully elucidated. The incidence of such cases remains fairly poorly defined, apart from the GI multifocal lymphomas that constitute $4 \sim 7 \%$ of all cases $(8,9)$.

The majority of these cases show gastric or intestinal involvement, with or without the involvement of other extranodal sites. The coupling of the lung with another extranodal site is relatively common. The remaining multifocal cases include patients with head and neck lymphoma, bone lymphoma and testicular lymphoma. The reason for multifocal extranodal lymphomas or the preferential involvement of specific extranodal sites at recurrence remains to be investigated, but it is likely that this is closely associated with the homing process that's regulated by homing receptors, or by the lymphoid cells 
and ligands on the endothelial venules (10). This tropism for specific sites has been particularly noted in the tonsils and stomach, testes $(11,12)$, and nasopharynx, in addition to the skin and bones $(10,13)$.

It appears that B-cell lymphomas frequently require stimulation involving the antigen receptor or other receptors on the surfaces of the lymphoma cells. It may be that the stimulus is the same for different origin sites of B-cell lymphoma. The genetic events that occur in germinal cells and give rise to B-cell lymphoma do not exist during the development of T-cell lymphocytes. This may be why $\mathrm{T}$ cells give rise to lymphomas one-tenth to one-twentieth as frequently as do the B cells (14).

In this case report, we have described an unusual case of extranodal multi-organ DLBL involving the femur, tibia and fibula with soft tissue extension, and this malady was also simultaneously detected in the stomach. The simultaneous detection of multiple extranodal involvement is quite uncommon. NHL of the bone is a rare condition. In many cases, its diagnosis is delayed as the result of the nonspecific clinical signs and equivocal radiographs. CT and FDG-PET scanning revealed, in this case, a rapidly extensive spread to the nodes and bones. The patient was diagnosed with stage IV DLBL, IPI 3, and he achieved good partial remission after chemotherapy and autologous PBSCT.

\section{REFERENCES}

1. Møller MB, Pedersen NT, Christensen BE. Diffuse large B-cell lymphoma: clinical implications of extranodal versus nodal presentation--a population-based study of 1575 cases. $\mathrm{Br}$ J Haematol. 2004;124:151-9.

2. Kadin ME, Carpenter C. Systemic and primary cutaneous anaplastic large cell lymphomas. Semin Hematol. 2003;40:24456.

3. Widder S, Pasieka JL. Primary thyroid lymphomas. Curr Treat Options Oncol. 2004;5:307-13.
4. Eom DW, Huh JR, Kang YK, Lee YS, Yu E. Clinicopathological features of eight Korean cases of primary hepatic lymphoma. Pathol Int. 2004;54:830-6.

5. Economopoulos T, Papageorgiou S, Rontogianni D, Kaloutsi V, Fountzilas G, Tsatalas C, et al. Multifocal extranodal nonHodgkin lymphoma: a clinicopathological study of 37 cases in Greece, a Hellenic Cooperative Oncology Group Study. Oncologist. 2005;10:734-8.

6. d'Amore F, Christensen BE, Brincker H, Pedersen NT, Thorling K, Hastrup J, et al. Clinicopathological features and prognostic factors in extranodal non-Hodgkin lymphomas. Danish LYFO Study Group. Eur J Cancer. 1991;27:1201-8.

7. Isaacson PG, Norton AJ. Multifocal extranodal lymphoma. In: Isaacson PG, Norton AJ, editors. Extranodal lymphomas. Edinburgh: Churchill Livingstone; 1994. p. 315-29.

8. Koch P, del Valle F, Berdel WE, Willich NA, Reers B, Hiddemann $\mathrm{W}$, et al. Anatomic and histologic distribution, clinical features, and survival data of 371 patients registered in the German Multicenter Study GIT NHL 01/92. J Clin Oncol. 2001;19:3861-73.

9. d'Amore F, Brincker H, Grønbaek K, Thorling K, Pedersen $M$, Jensen MK, et al. Non-Hodgkin's lymphoma of the gastrointestinal tract: a population-based analysis of incidence, geographic distribution, clinicopathologic presentation features, and prognosis. Danish Lymphoma Study Group. J Clin Oncol. 1994;12:1673-84.

10. Advani SH, Iyer RS, Gopal R, Nair CN, Saikia T, Dinshaw $\mathrm{KA}$, et al. Multifocal extranodal lymphomas: an expression of homing phenomenon. Oncology. 1990;47:334-8.

11. Economopoulos T, Asprou N, Stathakis N, Fountzilas G, Pavlidis N, Papaspyrou S, et al. Primary extranodal nonHodgkin's lymphoma of the head and neck. Oncology. 1992; 49:484-8.

12. Yuen A, Jacobs C. Lymphomas of the head and neck. Semin Oncol. 1999;26:338-45.

13. Shahab N, Doll DC. Testicular lymphoma. Semin Oncol. 1999;26:259-69.

14. Kuppers R, Klein U, Hansmann ML, Rajewsky K. Cellular origin of human B-cell lymphomas. N Engl J Med. 1999;341: $1520-9$. 